Rev. Bras. Saúde Prod. Anim., Salvador, v.16, n.3, p.617-631 jul./set.., 2015 http://www.rbspa.ufba.br

\title{
Estudo colaborativo para avaliação dos teores de matéria seca em alimentos
}

\author{
Collaborative study to evaluate the dry matter content in feeds
}

\author{
SOUZA, Marjorrie Augusto de ${ }^{1}$; DETMANN, Edenio ${ }^{2 *}$; VALADARES FILHO, \\ Sebastião de Campos ${ }^{2}$; FRANCO, Marcia de Oliveira ${ }^{2}$; ROCHA, Gabriel Cipriano ${ }^{2}$; \\ CABRAL, Luciano da Silva ${ }^{3}$
}

\author{
${ }^{1}$ Faculdade de Estudos Administrativos de Minas Gerais, Belo Horizonte, Minas Gerais, Brasil. \\ ${ }^{2}$ Universidade Federal de Viçosa, Departamento de Zootecnia, Viçosa, Minas Gerais, Brasil. \\ ${ }^{3}$ Universidade Federal do Mato Grosso, Departamento de Zootecnia e Extensão Rural, Cuiabá, Mato \\ Grosso, Brasil. \\ *Endereço para correspondência: detmann@ufv.br
}

\section{RESUMO}

Objetivou-se conduzir estudo colaborativo para avaliação do teor de matéria seca em alimentos em seis laboratórios de análise de alimentos de instituições integrantes do Instituto Nacional de Ciência e Tecnologia de Ciência Animal (INCTCA). Avaliaram-se seis alimentos: cana-de-açúcar, silagem de milho, capim-elefante, farelo de soja, polpa cítrica e mistura de milho grão com ureia (9:1). Solicitou-se a cada laboratório análises das amostras por onze dias, consecutivos ou não, sendo realizadas três repetições por amostra por dia de avaliação. Observou-se variação entre laboratórios, dias de análise e efeito de interação de material e laboratório. Avaliação comparativa das médias produzidas pelos laboratórios e pelo método de Karl Fischer indicou que 11,1\% das avaliações foram exatas. A variação dos resultados entre laboratórios correspondeu de 54,1 a $79,2 \%$ da variação aleatória total. Verificou-se que a repetibilidade representou de 6,24 a $26,45 \%$ da reprodutibilidade. Observou-se razões de Horwitz inferiores a 2 para os materiais avaliados. Percebeu-se que $52,8 \%$ das avaliações apresentaram variações das estimativas em função do dia no qual a análise foi realizada. Elevada variabilidade inter-laboratorial foi verificada por intermédio da avaliação dos questionários enviados aos laboratórios para elucidação dos principais procedimentos adotados, evidenciando possíveis causas da alta variação dos resultados.

Palavras-chave: análise de alimentos, estudos colaborativos, titulação de Karl Fischer, métodos de análises

\section{SUMMARY}

The objective was to conduct a collaborative study to evaluate the dry matter contents in feeds in six feed analysis laboratories from institutions linked to the National Institute of Science and Technology in Animal Science (INCT-CA). Six feeds were evaluated: sugarcane, corn silage, elephant grass, soybean meal, citrus pulp, and corn mixed with urea $(9: 1)$. It was asked to each laboratory that the samples were analyzed for eleven days, consecutive or not, with three replicates per sample per day of evaluation. There were variations among laboratories, days of analysis and interaction effect of material and laboratory. Comparative evaluation between the average contents produced by the laboratories and the Karl Fischer method indicated that $11.1 \%$ of the assessments were found accurate. The variation of results among laboratories accounted from 54.1 to $79.2 \%$ of the total random variation. It was found that the repeatability represented 6.24 to $26.45 \%$ of reproducibility. There are Horwitz ratios less than 2 for all materials tested. It was noticed that $52.8 \%$ of the assessments show variations of the estimates as a function of days in which analysis was performed. High interlaboratory variability was checked through the evaluation of questionnaires sent to laboratories to elucidate the main procedures used in each laboratory, which can possible explain the great variation of the estimates obtained here.

Keywords: analytical methods, collaborative study, feed analysis, Karl Fischer titration 
Rev. Bras. Saúde Prod. Anim., Salvador, v.16, n.3, p.617-631 jul./set.., 2015 http://www.rbspa.ufba.br

\section{INTRODUÇÃO}

O teor de umidade residual de uma amostra manejada em laboratório representa a umidade remanescente em um alimento úmido após sua desidratação prévia em estufas com ventilação forçada ou liofilizadores, ou a umidade total de alimentos com baixo teor de umidade, como grãos e farelos. Essa umidade é rotineiramente representada por seu complemento, denominado "amostra seca em estufa" (ASE), em virtude da maior facilidade dos cálculos posteriores para quantificação dos teores dos componentes químicos nas amostras. Para amostras com baixo teor de umidade, como fenos e concentrados, o teor de ASE de uma amostra corresponde ao seu teor de matéria seca (MS).

Em termos de rotina laboratorial, o correto conhecimento do teor de ASE nas amostras se mostra relevante, uma vez que estas são manejadas na base seca ao ar, ou seja, ainda contendo umidade. Desta forma, devido à impossibilidade de manejo de amostras totalmente secas, o teor de ASE é utilizado para correta expressão dos teores obtidos com base na MS da amostra. Contudo, por constituir denominador comum a todos os demais procedimentos laboratoriais, erros cometidos na quantificação dos teores de ASE tornam-se vícios ou erros sistemáticos em todas as demais avaliações, propagando-se, desta forma, ao entendimento global de todas as características do alimento (MERTENS, 2003).

Os teores de ASE de alimentos são normalmente obtidos no Brasil por intermédio da secagem em estufas isentas de ventilação em temperaturas iguais ou superiores à temperatura de ebulição da água (SILVA \& QUEIROZ, 2002). Contudo, diferentes binômios tempo $\times$ temperatura podem conduzir a diferentes resultados, com alta possibilidade de interação com amostras de diferentes origens e composições (THIEX \& RICHARDSON, 2003).

Desta forma, faz-se necessária a avaliação dos procedimentos laboratoriais e das estimativas de ASE produzidas em diferentes materiais e por diferentes laboratórios, buscando-se identificar métodos mais adequados e padronizar os procedimentos entre laboratórios de análise de alimentos no país.

Objetivou-se conduzir estudo colaborativo para avaliação do teor de ASE em amostras de alimentos em seis laboratórios de análise de alimentos de instituições integrantes do Instituto Nacional de Ciência e Tecnologia de Ciência Animal (INCT-CA).

\section{MATERIAL E MÉTODOS}

As avaliações foram conduzidas em seis laboratórios de análise de alimentos de instituições componentes do INCT-CA: Universidade Federal de Viçosa, Viçosa-MG; Escola de Veterinária da Universidade Federal de Minas Gerais, Belo Horizonte-MG; Universidade Federal de Lavras, Lavras-MG; Universidade Estadual de Santa Cruz, Ilhéus-BA; Universidade Estadual Paulista Júlio de Mesquita Filho, Jaboticabal-SP; e Universidade Federal de Mato Grosso, Cuiabá-MT; entre julho e agosto de 2009.

Foram avaliadas amostras de seis alimentos: cana-de-açúcar, silagem de milho, capim-elefante, farelo de soja, polpa cítrica e mistura de milho grão com ureia na proporção 9:1. As amostras de volumosos úmidos foram secas em estufa com ventilação forçada $\left(60^{\circ} \mathrm{C}\right)$ e, em conjunto com as demais 
Rev. Bras. Saúde Prod. Anim., Salvador, v.16, n.3, p.617-631 jul./set.., 2015 http://www.rbspa.ufba.br ISSN 15199940

amostras, processadas em moinho de facas $(1 \mathrm{~mm})$.

As amostras foram acondicionadas em sacos plásticos e enviadas sem identificação aos laboratórios. $\mathrm{Na}$ oportunidade do envio, solicitou-se a cada laboratório que as amostras fossem analisadas por 11 dias, consecutivos ou não, sendo realizadas três repetições por amostra por dia de avaliação.

$$
Y_{i j k l}=\mu+L_{i}+M_{j}+L M_{i j}+D_{(i j) k}+\varepsilon_{(i j k) l}
$$

em que: $\mathrm{Y}_{\mathrm{ijkl}}=$ teor de $\operatorname{ASE}(\%)$ obtida no laboratório i, no material $\mathrm{j}$, na repetição 1 avaliada no dia de análise $\mathrm{k}$; $\mu=$ constante geral; $\mathrm{L}_{\mathrm{i}}=$ efeito do laboratório $\mathrm{i}$ (aleatório); $\mathrm{M}_{\mathrm{j}}=$ efeito do material $\mathrm{j}$ (fixo); $\mathrm{LM}_{\mathrm{ij}}=$ efeito de interação do laboratório $\mathrm{i}$ e material $\mathrm{j}$ (aleatório); $\mathrm{D}_{(\mathrm{ij}) \mathrm{k}}=$ efeito do dia de análise $\mathrm{k}$, aninhado à combinação entre
Em conjunto com as amostras, enviouse questionário para elucidação dos principais procedimentos e parâmetros analíticos quantitativos e qualitativos adotados em cada laboratório.

Após o recebimento dos dados, os mesmos foram analisados de forma global de acordo com o modelo:

Tabela 1. Esperanças de quadrados médios para o modelo destinado à análise global dos dados (Equação 1)

\begin{tabular}{ll}
\hline Fonte de Variação $^{1}$ & \multicolumn{1}{c}{$\mathrm{E}(\mathrm{QM})^{2}$} \\
\hline $\mathrm{L}$ & $\sigma_{\varepsilon}^{2}+\mathrm{k}_{1} \times \sigma_{\mathrm{D} / \mathrm{L} \times \mathrm{M}}^{2}+\mathrm{k}_{2} \times \sigma_{\mathrm{L} \times \mathrm{M}}^{2}+\mathrm{k}_{4} \times \sigma_{\mathrm{L}}^{2}$ \\
$\mathrm{M}$ & $\sigma_{\varepsilon}^{2}+\mathrm{k}_{1} \times \sigma_{\mathrm{D} / \mathrm{L} \times \mathrm{M}}+\mathrm{k}_{2} \times \sigma_{\mathrm{L} \times \mathrm{M}}^{2}+\mathrm{k}_{3} \times \phi_{\mathrm{M}}$ \\
$\mathrm{L} \times \mathrm{M}$ & $\sigma_{\varepsilon}^{2}+\mathrm{k}_{1} \times \sigma_{\mathrm{D} / \mathrm{L} \times \mathrm{M}}+\mathrm{k}_{2} \times \sigma_{\mathrm{L} \times \mathrm{M}}^{2}$ \\
$\mathrm{D} / \mathrm{L} \times \mathrm{M}$ & $\sigma_{\varepsilon}^{2}+\mathrm{k}_{1} \times \sigma_{\mathrm{D} / \mathrm{L} \times \mathrm{M}}$ \\
Resíduo & $\sigma_{\varepsilon}^{2}$ \\
\hline
\end{tabular}

${ }^{1} \mathrm{~L}=$ laboratório; $\mathrm{M}=$ material; $\mathrm{L} \times \mathrm{M}=$ interação laboratório $\times$ material; $\mathrm{D} / \mathrm{L} \times \mathrm{M}=$ dia aninhado às combinações entre laboratório e material.

${ }^{2} \sigma_{\varepsilon}^{2}, \sigma_{\mathrm{D} / \mathrm{L} \times \mathrm{M}}^{2}, \sigma_{\mathrm{L} \times \mathrm{M}}^{2}, \sigma_{\mathrm{L}}^{2}=$ variâncias associadas aos efeitos do erro (repetibilidade), de dia aninhado às combinações entre laboratório e material, da interação de laboratório e material e de laboratório, respectivamente; $\phi_{\mathrm{M}}=$ função quadrática associada ao efeito fixo de material.

As observações classificadas como outliers foram eliminadas do conjunto de dados, sendo estas definidas quando seu desvio em relação à média das avaliações do material, dentro do laboratório, superasse em três vezes o valor do desvio padrão residual. o laboratório i e o material j (aleatório); e $\varepsilon_{(\mathrm{ijk}) 1}=$ erro aleatório, associado a cada mensuração, pressuposto NID $\left(0 ; \sigma_{\varepsilon}^{2}\right)$. Por intermédio do método dos momentos (BARBIN, 1993), definiramse as esperanças de quadrados médios da análise de variância representada pela equação (1) (Tabela 1). 
Rev. Bras. Saúde Prod. Anim., Salvador, v.16, n.3, p.617-631 jul./set.., 2015 http://www.rbspa.ufba.br ISSN 15199940

em que: $\mathrm{r}=$ repetibilidade; $\mathrm{e} \mathrm{R}=$ reprodutibilidade.

Quando o efeito de interação de material analisado e laboratório foi significativo, procedeu-se a nova avaliação, na qual considerou-se cada material isoladamente. Esclarece-se que na presença de interação, tanto a reprodutibilidade como a repetibilidade estimadas a partir dos componentes de variância obtidos na equação (1) não podem ser utilizadas para representar o comportamento de cada alimento em geral. Como são avaliados efeitos aleatórios não é possível realizar o desdobramento em um único modelo. Cabe ressaltar, que efeitos aleatórios não constituem parâmetros da população, mas sim constituem variáveis aleatórias contínuas. Desta forma, um componente de variância comum para laboratório não poderá ser usado para a avaliação de cada um dos alimentos. Como o desdobramento em um único modelo é impossível neste caso, a estimação de componentes de variâncias individuais só é possível com o fracionamento do banco de dados. Desta forma, o desdobramento foi realizado utilizando-se um modelo para avaliação da variação de cada material considerando-se os diferentes laboratórios:

$Y_{i j k}=\mu+L_{i}+D_{(i) j}+\varepsilon_{(i j) k}$

em que: $\mathrm{Y}_{\mathrm{ijk}}=$ teor de ASE (\%) obtida no laboratório $\mathrm{i}$, na repetição $\mathrm{k}$ avaliada no dia de análise $\mathrm{j} ; \mu=$ constante geral; $\mathrm{L}_{\mathrm{i}}=$ efeito do laboratório i (aleatório); $\mathrm{D}_{(\mathrm{i}) \mathrm{j}}=$ efeito do dia de análise $\mathrm{j}$ aninhado ao laboratório i (aleatório); e $\varepsilon_{(\mathrm{ij}) \mathrm{k}}=$ erro aleatório, associado a cada mensuração, pressuposto NID $\left(0 ; \sigma_{\varepsilon}^{2}\right)$.

As esperanças de quadrados médios para o modelo descrito em (4) são mostradas na Tabela 2. As estimativas de repetibilidade e reprodutibilidade para cada material foram obtidas de forma similar ao descrito nas equações (2) e (3).

Tabela 2. Esperanças de quadrados médios para o modelo destinado à análise de cada material em função dos diferentes laboratórios (Equação 4)

\begin{tabular}{cc}
\hline Fonte de Variação $^{1}$ & \multicolumn{1}{c}{$\mathrm{E}(\mathrm{QM})^{2}$} \\
\hline $\mathrm{L}$ & $\sigma_{\varepsilon}^{2}+\mathrm{k}_{1} \times \sigma_{\mathrm{D} / \mathrm{L}}+\mathrm{k}_{2} \times \sigma^{2} \mathrm{~L}$ \\
$\mathrm{D} / \mathrm{L}$ & $\sigma_{\varepsilon}^{2}+\mathrm{k}_{1} \times \sigma_{\mathrm{D} / \mathrm{L}}^{2}$ \\
Resíduo & $\sigma_{\varepsilon}^{2}$ \\
${ }^{1} \mathrm{~L}=$ laboratório; $\mathrm{D} / \mathrm{L}=$ dia aninhado ao laboratório. \\
${ }^{2} \sigma_{\varepsilon}^{2}, \sigma_{\mathrm{D} / \mathrm{L}}, \sigma_{\mathrm{L}}^{2}=$ variâncias associadas aos efeitos do erro (repetibilidade), de dia aninhado ao laboratório \\
e de laboratório, respectivamente.
\end{tabular}

Para a situação descrita na Tabela 2, procedeu-se à avaliação das estimativas da reprodutibilidade esperada e da razão de Horwitz (HORWITZ et al., 1990) por intermédio das equações:

$$
\begin{aligned}
& R p=\frac{\hat{\sigma}_{R}}{\bar{X}} \quad \text { (5); } \\
& \hat{\sigma}_{R}=\sqrt{R} \quad \text { (6); }
\end{aligned}
$$

$$
\begin{aligned}
& \text { Rpe }=2 \times \exp (1-0,5 \times \log c) \quad(7) ; \\
& R H=\frac{R p}{R p e}
\end{aligned}
$$

em que: $\mathrm{Rp}=$ reprodutibilidade padronizada em função da média; Rpe = reprodutibilidade esperada padronizada em função da média; c = concentração média de $\operatorname{ASE}(\mathrm{g} / \mathrm{g})$; e $\mathrm{RH}=$ razão de Horwitz. 
Rev. Bras. Saúde Prod. Anim., Salvador, v.16, n.3, p.617-631 jul./set.., 2015 http://www.rbspa.ufba.br

Em adição, quando o efeito de dia de análise foi significativo, procedeu-so ao desdobramento das avaliações conforme explicações apresentadas anteriormente, com a avaliação do comportamento de cada material em cada laboratório por intermédio do modelo:

$Y_{i j}=\mu+D_{i}+\varepsilon_{(i) j}$

em que: $\mathrm{Y}_{\mathrm{ij}}=$ teor de ASE (\%) obtida na repetição $\mathrm{j}$ avaliada no dia de análise $i ; \mu$ $=$ constante geral; $\mathrm{D}_{\mathrm{i}}=$ efeito do dia de análise i (aleatório); e $\varepsilon_{(\mathrm{i}) \mathrm{j}}=$ erro aleatório, associado a cada mensuração, pressuposto NID $\left(0 ; \sigma_{\varepsilon}^{2}\right)$.

Por intermédio do método dos momentos (BARBIN, 1993), definiramse as esperanças de quadrados médios associadas ao modelo descrito em (9) (Tabela 3).

A variação aleatória total associada ao modelo descrito em (9) foi dada por:

$\hat{\sigma}_{T}^{2}=\hat{\sigma}_{D}^{2}+\hat{\sigma}_{\varepsilon}^{2}(10)$.
Para verificação da exatidão dos resultados, todas as amostras foram avaliadas quanto ao teor de umidade residual por intermédio do método químico de Karl Fischer (BRUTTEL \& SCHLINK, 2006), utilizando-se equipamento $870 \mathrm{KF}$ Titrino Plus, Metrohm.

Todos os procedimentos estatísticos foram conduzidos por intermédio do programa Statisical Analysis System, versão 9.3 (SAS, 2012), adotando-se 0,001 como nível crítico de probabilidade para o erro tipo I. As comparações entre médias fornecidas por diferentes laboratórios foram conduzidas utilizando-se o critério de Scheffé. Por outro lado, a comparação entre estas médias e o valor esperado produzido pelo método padrão foram realizadas utilizando-se as propriedades de intervalos de confiança $(1-\alpha=$ $0,999)$.

Tabela 3. Esperanças de quadrados médios para o modelo destinado à análise de cada material em cada laboratório em função dos dias de análise (Equação 9)

\begin{tabular}{lc}
\hline Fonte de Variação $^{1}$ & $\mathrm{E}(\mathrm{QM})^{2}$ \\
\hline $\mathrm{D}$ & $\sigma_{\varepsilon}^{2}+\mathrm{k}_{1} \times \sigma^{2}{ }_{\mathrm{D}}$ \\
Resíduo & $\sigma_{\varepsilon}^{2}$ \\
\hline${ }^{1} \mathrm{D}=$ dia; ${ }^{2} \sigma_{\varepsilon}^{2}, \sigma^{2}{ }_{\mathrm{D}}=$ variâncias associadas aos efeitos do erro e de dia de análise, respectivamente.
\end{tabular}

\section{RESULTADOS E DISCUSSÃO}

Observou se que todos os componentes de variância do modelo global (Equação 1) foram significativos $(\mathrm{P}<0,001)$. Isto indica que, além das diferenças entre laboratórios, os teores estimados de ASE se mostram dependentes do dia em que a análise foi realizada e que materiais diferentes produziram estimativas diferentes em função do laboratório no qual a análise foi realizada (Tabela 4).
De forma geral, assume-se como não aceitável a ocorrência de efeito de interação de material e laboratório (MERTENS, 2003). A ocorrência deste efeito indica que os possíveis erros sistemáticos imputados a um dado material em determinado laboratório serão distintos daqueles observados em outro laboratório. A isto, se adiciona o fato de que os erros sistemáticos imputados por um laboratório se manifestarão de forma diferenciada em função do material analisado. Desta forma, como ressaltado anteriormente, 
Rev. Bras. Saúde Prod. Anim., Salvador, v.16, n.3, p.617-631 jul./set.., 2015 http://www.rbspa.ufba.br ISSN 15199940

as composições químicas das amostras com base na MS poderão ser expressas de forma diferente por diferentes laboratórios não por diferenças na composição química em si, mas por diferenças nas estimativas de ASE dos materiais. Isto atribui elemento de confundimento aos cotejamentos das características químicas de alimentos e outros materiais, como fezes, sobras e digestas; o que, consequentemente, pode comprometer a comparação entre características obtidas em ensaios de digestão e metabolismo.

Tabela 4. Estimativas de componentes de variância do modelo global (Equação 1)

\begin{tabular}{lccc}
\hline Item & Estimativa & Valor relativo (\%) & Valor-P \\
\hline$\sigma^{2}{ }_{\mathrm{L}}$ & 1,0827 & 44,1 & $<0,0001$ \\
$\sigma_{\mathrm{L} \times \mathrm{M}}$ & 0,6031 & 24,6 & $<0,0001$ \\
$\sigma_{\mathrm{D} / \mathrm{L} \times \mathrm{M}}^{2}$ & 0,4933 & 20,1 & $<0,0001$ \\
Repetibilidade $\left(\sigma_{\varepsilon}^{2}\right)$ & 0,2746 & 11,2 & - \\
Variância Total & 2,4537 & 100,0 & - \\
Reprodutibilidade & 1,3573 & - & - \\
$\mathrm{r} / \mathrm{R}^{1}$ & 0,2023 & - & - \\
\hline
\end{tabular}

${ }^{1}$ Razão repetibilidade/reprodutibilidade.

A interação de material e laboratório é corroborada pela avaliação comparativa das médias obtidas nos diferentes alimentos, na qual se observou alta variabilidade nas diferenças entre laboratórios, não havendo padrão definido nos perfis de comparações múltiplas. Verificou-se que as diferenças entre laboratórios variaram de 1,91 ponto percentual para o farelo de soja a 5,21 pontos percentuais para a silagem de milho (Tabela 5).

Tabela 5. Comparação entre as médias produzidas pelos diferentes laboratórios para cana-de-açúcar, silagem de milho (SM), capim-elefante (CE), farelo de soja (FS), milho + ureia (MUR) e polpa cítrica (PC)

\begin{tabular}{ccccccc}
\hline \multirow{2}{*}{ Laboratório } & \multicolumn{7}{c}{ Material $^{12}$} \\
\cline { 2 - 7 } & Cana & SM & CE & FS & MUR & PC \\
\hline Padrão $^{3}$ & 90,08 & 89,89 & 89,22 & 90,36 & 89,85 & 89,14 \\
\hline 1 & $93,46^{\mathrm{b} *}$ & $92,82^{\mathrm{ab} *}$ & $92,62^{\mathrm{c} *}$ & $88,29^{\mathrm{bc} *}$ & $89,15^{\mathrm{b}}$ & $90,42^{\mathrm{ab} *}$ \\
2 & $93,64^{\mathrm{b} *}$ & $89,79^{\mathrm{c}}$ & $92,98^{\mathrm{bc} *}$ & $88,56^{\mathrm{b} *}$ & $89,80^{\mathrm{b}}$ & $91,37^{\mathrm{a} *}$ \\
3 & $93,27^{\mathrm{b} *}$ & $92,59^{\mathrm{ab} *}$ & $94,25^{\mathrm{b} *}$ & $88,10^{\mathrm{bc} *}$ & $89,05^{\mathrm{b} *}$ & $89,20^{\mathrm{bc}}$ \\
4 & $91,52^{\mathrm{c} *}$ & $91,69^{\mathrm{bc} *}$ & $92,35^{\mathrm{c} *}$ & $87,50^{\mathrm{c} *}$ & $87,19^{\mathrm{c}} *$ & $88,10^{\mathrm{c} *}$ \\
5 & $93,34^{\mathrm{b} *}$ & $93,26^{\mathrm{ab} *}$ & $93,23^{\mathrm{bc} *}$ & $88,34^{\mathrm{b} *}$ & $89,10^{\mathrm{b} *}$ & $91,06^{\mathrm{a} *}$ \\
6 & $95,50^{\mathrm{a} *}$ & $95,00^{\mathrm{a} *}$ & $95,93^{\mathrm{a} *}$ & $89,41^{\mathrm{a} *}$ & $91,30^{\mathrm{a} *}$ & $91,60^{\mathrm{a} *}$ \\
EPM $^{\text {aTM }}$ & 0,191 & 0,397 & 0,190 & 0,118 & 0,185 & 0,203 \\
ATM $^{4}$ & 3,98 & 5,21 & 3,58 & 1,91 & 4,11 & 3,50 \\
\hline
\end{tabular}

${ }^{\mathrm{T}}$ Médias na coluna, seguidas por letras diferentes, são diferentes pelo teste de Scheffé $(\mathrm{P}<0,001)$.

${ }^{2}$ Médias seguidas por $(*)$ apresentam vício significativo em relação ao método padrão utilizando-se as propriedades do intervalo de confiança $[(1-\alpha)=0,999]$.

${ }^{3}$ Valor obtido com a titulação de Karl Fischer.

${ }^{4}$ ATM, amplitude total entre médias (diferença entre a maior e menor média de laboratórios). 
Rev. Bras. Saúde Prod. Anim., Salvador, v.16, n.3, p.617-631 jul./set.., 2015 http://www.rbspa.ufba.br ISSN 15199940

Adicionalmente, a avaliação comparativa das médias produzidas pelos diferentes laboratórios e pelo método de Karl Fischer (padrão) indicou que, das 36 avaliações, apenas $4(11,1 \%)$ foram consideradas exatas (Tabela 5). Em outras palavras, em $88,9 \%$ das avaliações, erros sistemáticos advindos de falhas na avaliação da umidade residual seriam imputados sobre a composição química dos alimentos com base na MS.

A maioria das análises químicas ou biológicas em alimentos é conduzida em amostras secas ao ar. Contudo, as concentrações dos diferentes componentes químicos devem ser expressas com base na MS, o que permite comparações diretas da concentração de componentes químicos sem $\mathrm{O}$ confundimento causado pela umidade do material (MERTENS, 2003). Como a transformação da massa de amostra seca ao ar em massa de MS é feita utilizandose a ASE, erros na quantificação da ASE podem resultar em falsas diferenças na concentração de um componente químico do alimento com base na MS.

Exemplo dos efeitos sobre a composição química dos alimentos advindos de diferenças nas estimativas de ASE é apresentado na Tabela 6. Neste caso, assumiu-se composição hipotética da silagem de milho com base na matéria seca ao ar e contrastouse a partir dos valores extremos de ASE, os quais foram obtidos com os laboratórios 2 e 6 (Tabela 5).

Tabela 6. Exemplo teórico da influência das variações do teor de ASE na composição química de alimento volumoso

\begin{tabular}{lcccc}
\hline \multirow{2}{*}{ Componente $^{1}$} & \multirow{2}{*}{${\text { Composição teórica }(\%)^{2}}^{*}$} & \multicolumn{2}{c}{ Laboratório } & \multirow{2}{*}{ Diferencial (6-2; \%) } \\
\cline { 3 - 4 } MS & - & 89,79 & 95,00 & $+5,8$ \\
PB & 8 & 8,91 & 8,42 & $-5,5$ \\
EE & 3 & 3,34 & 3,16 & $-5,4$ \\
FDNcp & 55 & 61,25 & 57,89 & $-5,5$ \\
MM & 5 & 5,57 & 5,26 & $-5,6$ \\
$\mathrm{CNF}^{(3)}$ & - & 20,93 & 25,27 & $+20,7$ \\
\hline
\end{tabular}

${ }^{\mathrm{I}} \mathrm{MS}=$ matéria seca; $\mathrm{PB}=$ proteína bruta; $\mathrm{EE}=$ extrato etéreo; $\mathrm{FDNcp}=$ fibra em detergente neutro corrigida para cinzas e proteína; $\mathrm{MM}=$ matéria mineral; $\mathrm{CNF}=$ carboidratos não fibrosos.

${ }^{2}$ Composição assumida hipoteticamente com base na matéria seca ao ar para uma amostra de silagem de milho.

${ }^{3} \mathrm{CNF}=100-(\mathrm{PB}+\mathrm{EE}+\mathrm{FDNcp}+\mathrm{MM})$.

Observa-se que as diferenças entre os teores de ASE se projetam em magnitudes similares, mas em direções opostas, sobre os componentes químicos analisados diretamente (Tabela 6). Contudo, os vícios imputados sobre estes componentes são potencializados sobre o teor do componente químico estimado por diferença, neste caso carboidratos não fibrosos, uma vez que este absorverá todos os erros associados com os teores dos componentes químicos analisados diretamente (DETMANN \& VALADARES FILHO, 2010).

Em algumas situações, vícios ou erros sistemáticos podem ser tolerados, pois, sendo estes constantes, fatores de correção podem ser utilizados (HORWITZ, 1982). Contudo, o efeito 
de interação verificado neste trabalho indica que a magnitude dos vícios varia em função da combinação entre laboratório e material, tornando inviável qualquer tipo de correção sobre os dados.
Analisando-se os materiais isoladamente, observou-se grande variação na reprodutibilidade (Figura 1), o que parece mais uma vez corroborar o efeito de interação de laboratório e material (Tabelas 4 e 5).

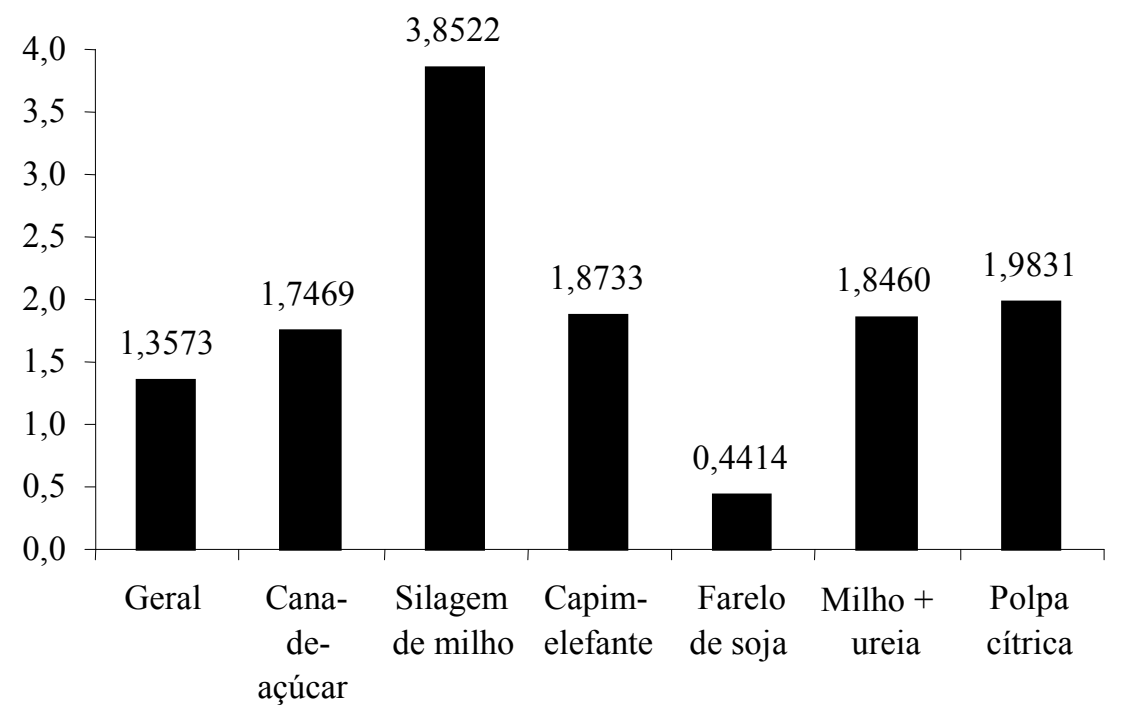

Figura 1. Reprodutibilidade $\left[(\%)^{2}\right]$ para os diferentes materiais analisados

$\mathrm{Na}$ avaliação individual de cada material, observou-se que a variação dos resultados entre laboratórios correspondeu de 54,1 a $79,2 \%$ da variação aleatória total. Adicionalmente, verificou-se que a repetibilidade (variação entre repetições) representou de 6,24 a $26,45 \%$ da reprodutibilidade (Tabelas 7 e 8 ). O comportamento destes percentuais reitera que diferenças entre laboratórios correspondem ao fator determinante das diferenças entre os teores de ASE.

Contudo, a avaliação da reprodutibilidade, a qual é dada pela soma da repetibilidade e da variação entre laboratórios apresentou-se em patamares teoricamente aceitáveis, pois se observou razões de Horwitz (RH) inferiores a 2 para todos os materiais avaliados (Tabelas 7 e 8). Este parâmetro é dado pela razão entre a reprodutibilidade observada e a reprodutibilidade que deveria ser esperada considerando-se a concentração média do componente analisado (HORWITZ et al., 1990).

Com poucas exceções, RH superiores a 2 sugerem que o método avaliado é inaceitável com respeito à sua reprodutibilidade (MERTENS, 2003). Caso este critério seja adotado neste estudo, poderia se concluir que as avaliações seriam coerentes para os diferentes materiais e apresentariam bom nível de reprodutibilidade. Contudo, estas características não correspondem aos resultados aqui obtidos (Tabelas 4 e 5).

Deve ser percebido que neste trabalho não se procedeu à avaliação de método em si, uma vez que os procedimentos não foram sugeridos ou padronizados. As amostras foram analisadas de acordo com os procedimentos rotineiramente 
Rev. Bras. Saúde Prod. Anim., Salvador, v.16, n.3, p.617-631 jul./set.., 2015 http://www.rbspa.ufba.br ISSN 15199940

aplicados em cada laboratório participante. Assim, a definição apresentado por Mertens (2003) não poderia ser plenamente aplicada às condições deste estudo.

Adicionalmente, a partir dos dados expressos nas Tabelas 7 e 8 , estima-se que as repetibilidades padronizadas (expressas como percentuais das médias) foram de 0,$44 ; 1,09 ; 0,38 ; 0,29 ; 0,38 \mathrm{e}$ 0,41\% para cana-de-açúcar, silagem de milho, capim-elefante, farelo de soja, milho + ureia e polpa cítrica, respectivamente. Estas estimativas indicam baixos valores relativos da variação entre repetições. Logo, sendo a reprodutibilidade dada pela soma da variação entre repetições e entre laboratórios, os baixos valores de $\mathrm{RH}$ (Tabelas 7 e 8) parecem ter sido causados pela baixa variação entre repetições. Logo, embora aparentemente em patamares adequados, os valores de $\mathrm{RH}$ não expressaram, nas condições deste estudo, a elevada variação entre laboratórios.

Tabela 7. Estimativas de componentes de variância do modelo destinado à avaliação individual de alimentos volumosos (Equação 4)

\begin{tabular}{lccc}
\hline Item & Estimativa & Valor relativo (\%) & Valor-P \\
\hline$\sigma_{\mathrm{L}}^{2}$ & \multicolumn{3}{c}{ Cana-de-açúcar } \\
$\sigma_{\mathrm{D} / \mathrm{L}}$ & 1,5773 & 75,4 & $<0,0001$ \\
Repetibilidade $\left(\sigma_{\varepsilon}^{2}\right)$ & 0,3456 & 16,5 & $<0,0001$ \\
Variância Total & 0,1696 & 8,1 & - \\
Reprodutibilidade & 2,0925 & 100,0 & - \\
$\mathrm{r} / \mathrm{R}^{1}$ & 1,7469 & - & - \\
$\mathrm{RH}$ & 0,0971 & - & - \\
Média & 0,6973 & - & - \\
\hline & 93,45 & - & - \\
\hline$\sigma_{\mathrm{L}}^{2}$ & & Silagem de milho & $<0,0001$ \\
$\sigma_{\mathrm{D} / \mathrm{L}}$ & 54,1 & $<0,0001$ \\
Repetibilidade $\left(\sigma_{\varepsilon}^{2}\right)$ & 2,8333 & 26,5 & - \\
Variância Total & 1,3906 & 19,4 & - \\
Reprodutibilidade & 1,0189 & 100 & - \\
$\mathrm{r} / \mathrm{R}^{1}$ & 5,2428 & - & - \\
$\mathrm{RH}$ & 3,8522 & - & - \\
Média & 0,2645 & - & - \\
\hline & 1,0429 & - & $<0,0001$ \\
\hline$\sigma_{\mathrm{L}}^{2}$ & 92,53 & Capim-elefante & $<0,0001$ \\
$\sigma_{\mathrm{D} / \mathrm{L}}$ & & 78,3 & - \\
Repetibilidade $\left(\sigma_{\varepsilon}^{2}\right)$ & 1,7462 & 16,0 & - \\
Variância Total & 0,3568 & 5,7 & - \\
Reprodutibilidade & 0,1271 & 100,0 & - \\
$\mathrm{r} / \mathrm{R}^{1}$ & 2,2301 & - & - \\
$\mathrm{RH}$ & 1,8733 & - & - \\
Média & 0,0678 & - &
\end{tabular}


Rev. Bras. Saúde Prod. Anim., Salvador, v.16, n.3, p.617-631 jul./set.., 2015 http://www.rbspa.ufba.br ISSN 15199940

Tabela 8. Estimativas de componentes de variância do modelo destinado à avaliação individual de alimentos concentrados (Equação 4)

\begin{tabular}{|c|c|c|c|}
\hline Item & Estimativa & Valor relativo (\%) & Valor-P \\
\hline & \multicolumn{3}{|c|}{ Farelo de soja } \\
\hline$\sigma_{L}^{2}$ & 0,3759 & 65,7 & $<0,0001$ \\
\hline$\sigma^{2} \mathrm{D} / \mathrm{L}$ & 0,1312 & 22,9 & $<0,0001$ \\
\hline Repetibilidade $\left(\sigma_{\varepsilon}^{2}\right)$ & 0,0655 & 11,4 & - \\
\hline Variância Total & 0,5726 & 100,0 & - \\
\hline Reprodutibilidade & 0,4414 & - & - \\
\hline $\mathrm{r} / \mathrm{R}^{1}$ & 0,1484 & - & - \\
\hline RH & 0,3660 & - & - \\
\hline \multirow[t]{2}{*}{ Média } & 88,37 & - & - \\
\hline & \multicolumn{3}{|c|}{ Milho + ureia } \\
\hline$\sigma_{L}^{2}$ & 1,7309 & 79,2 & $<0,0001$ \\
\hline$\sigma_{\mathrm{D} / \mathrm{L}}^{2}$ & 0,3392 & 15,5 & $<0,0001$ \\
\hline Repetibilidade $\left(\sigma_{\varepsilon}^{2}\right)$ & 0,1151 & 5,3 & - \\
\hline Variância Total & 2,1852 & 100,0 & - \\
\hline Reprodutibilidade & 1,8460 & - & - \\
\hline $\mathrm{r} / \mathrm{R}^{1}$ & 0,0624 & - & - \\
\hline RH & 0,7425 & - & - \\
\hline \multirow[t]{2}{*}{ Média } & 89,26 & - & - \\
\hline & \multicolumn{3}{|c|}{ Polpa cítrica } \\
\hline$\sigma_{\mathrm{L}}^{2}$ & 1,8429 & 77,1 & $<0,0001$ \\
\hline$\sigma_{\mathrm{D} / \mathrm{L}}^{2}$ & 0,4063 & 17,0 & $<0,0001$ \\
\hline Repetibilidade $\left(\sigma_{\varepsilon}^{2}\right)$ & 0,1402 & 5,9 & - \\
\hline Variância Total & 2,3894 & 100 & - \\
\hline Reprodutibilidade & 1,9831 & - & - \\
\hline $\mathrm{r} / \mathrm{R}^{1}$ & 0,0707 & - & - \\
\hline RH & 0,7627 & - & - \\
\hline Média & 90,29 & - & - \\
\hline
\end{tabular}

Considerando-se as informações constantes nas Tabelas 7 e 8 , percebe-se que, para todos os materiais, ocorreu efeito significativo do dia de análise $(\mathrm{P}<0,001)$ sobre as estimativas de ASE. A avaliação isolada deste efeito para cada material analisado em cada laboratório indicou que $52,8 \%$ das avaliações apresentaram variações significativas $(\mathrm{P}<0,001)$ das estimativas de ASE em função do dia no qual a análise foi realizada (Tabela 9).

Tabela 9. Nível descritivo de probabilidade para o erro tipo I associada à variação entre dias de análises para cana-de-açúcar, silagem de milho (SM), capim-elefante (CE), farelo de soja (FS), milho + ureia (MUR) e polpa cítrica (PC)

\begin{tabular}{ccccccc}
\hline \multirow{2}{*}{ Laboratório } & \multicolumn{7}{c}{ Material } \\
\cline { 2 - 6 } & Cana & SM & CE & FS & MUR & PC \\
\hline 1 & $<0,0001$ & $<0,0001$ & $<0,0001$ & $<0,0001$ & $<0,0001$ & $<0,0001$ \\
2 & 0,0901 & 0,2992 & 0,3914 & 0,8097 & 0,1913 & 0,9523 \\
3 & 0,0156 & 0,1761 & $<0,0001$ & $<0,0001$ & $<0,0001$ & $<0,0001$ \\
4 & 0,0028 & $<0,0001$ & 0,0718 & 0,0009 & $<0,0001$ & $<0,0001$ \\
5 & 0,0282 & 0,0016 & $<0,0001$ & 0,0157 & 0,3655 & 0,0912 \\
6 & $<0,0001$ & $<0,0001$ & 0,1017 & 0,0012 & $<0,0001$ & $<0,0001$ \\
\hline
\end{tabular}


Rev. Bras. Saúde Prod. Anim., Salvador, v.16, n.3, p.617-631 jul./set.., 2015 http://www.rbspa.ufba.br ISSN 15199940

A variabilidade ao longo dos dias de análise implica que os resultados obtidos no processo analítico são diretamente dependentes deste fator, o que obviamente pode atribuir efeito de confundimento sobre a composição química do material analisado. Por lógica, evidencia-se que problema atribuído à falta de padronização dos procedimentos intra-laboratoriais somase à questão da alta variabilidade interlaboratorial sobre a confiabilidade dos resultados de ASE.

A avaliação individual da variação intralaboratorial (Figura 2), a qual constitui o fator determinante da repetibilidade, indica que a variação entre dias de análise pode representar de 0 (ex.: Figura 3; farelo se soja; Laboratório 2) a 99\% (ex.: Figura 3; silagem de milho; laboratório 1) da variação aleatória dos resultados.

Elevada variabilidade inter-laboratorial foi verificada por intermédio da avaliação dos questionários enviados aos diferentes laboratórios, o que evidencia possíveis causas da alta variação dos resultados observada neste estudo (Tabelas 4, 7 e 8). As características quantitativas do processo analítico não puderam ser consideradas constantes entre laboratórios (Tabela 10). Embora a temperatura de secagem tenha sido aproximadamente similar $\left(103\right.$ a $\left.105^{\circ} \mathrm{C}\right)$, o tempo de manutenção do material nas respectivas temperaturas mostrou-se altamente variado (4 a 16 horas). Sabendo-se que o processo físico de remoção da umidade do material é função do binômio tempo $\times$ temperatura, pode-se concluir que os laboratórios praticam essencialmente métodos diferentes.

Todos os laboratórios utilizam dessecador para resfriamento das amostras após o período de secagem, tendo a sílica gel como dessecante. Contudo, o número de alíquotas manejadas no dessecador é variável entre laboratórios (Tabela 10).
Dessecadores possuem seu próprio microambiente no qual as amostras e o dessecante entram em equilíbrio. Mesmo ainda capazes de absorver umidade, os dessecantes não absorvem toda a água da atmosfera interna do dessecador. Assim, a cada vez que o dessecador é aberto, as condições internas são alteradas, pois mais ar atmosférico é introduzido no ambiente. Consequentemente, introduz-se umidade, a qual é distribuída entre amostras e dessecante (VAN SOEST \& ROBERTSON, 1985).

Logo, quanto maior o número de alíquotas manejadas, maior a massa de umidade absorvida pelo conjunto amostra/pesa-filtro durante os procedimentos. Em alguns laboratórios, o número de pesa-filtros manejados em um único procedimento de pesagem pode ser considerado elevado (ex.: laboratórios 3 e 5), o que pode comprometer a acurácia de seus resultados e, por consequência, ampliar as variações inter e intra-laboratoriais.

Em termos de manejo do pesa-filtro, verificou-se que apenas dois laboratórios utilizam a tampa durante o processo de resfriamento no dessecador. Os demais laboratórios utilizam pesafiltros sem tampa ou recipientes diferentes, mas também isentos de tampa (ex.: cadinhos de porcelana). Considerando-se que a tampa atua como instrumento para redução da absorção de umidade pela amostra no interior do dessecador, adiciona-se novo elemento que pode ser parcialmente responsável pela baixa reprodutibilidade dos resultados obtidos neste trabalho.

Diferenças quanto à utilização de balanças também foram percebidas entre laboratórios. Em dois dos laboratórios não são utilizadas salas climatizadas de acordo com as necessidades de utilização de balanças analíticas. 

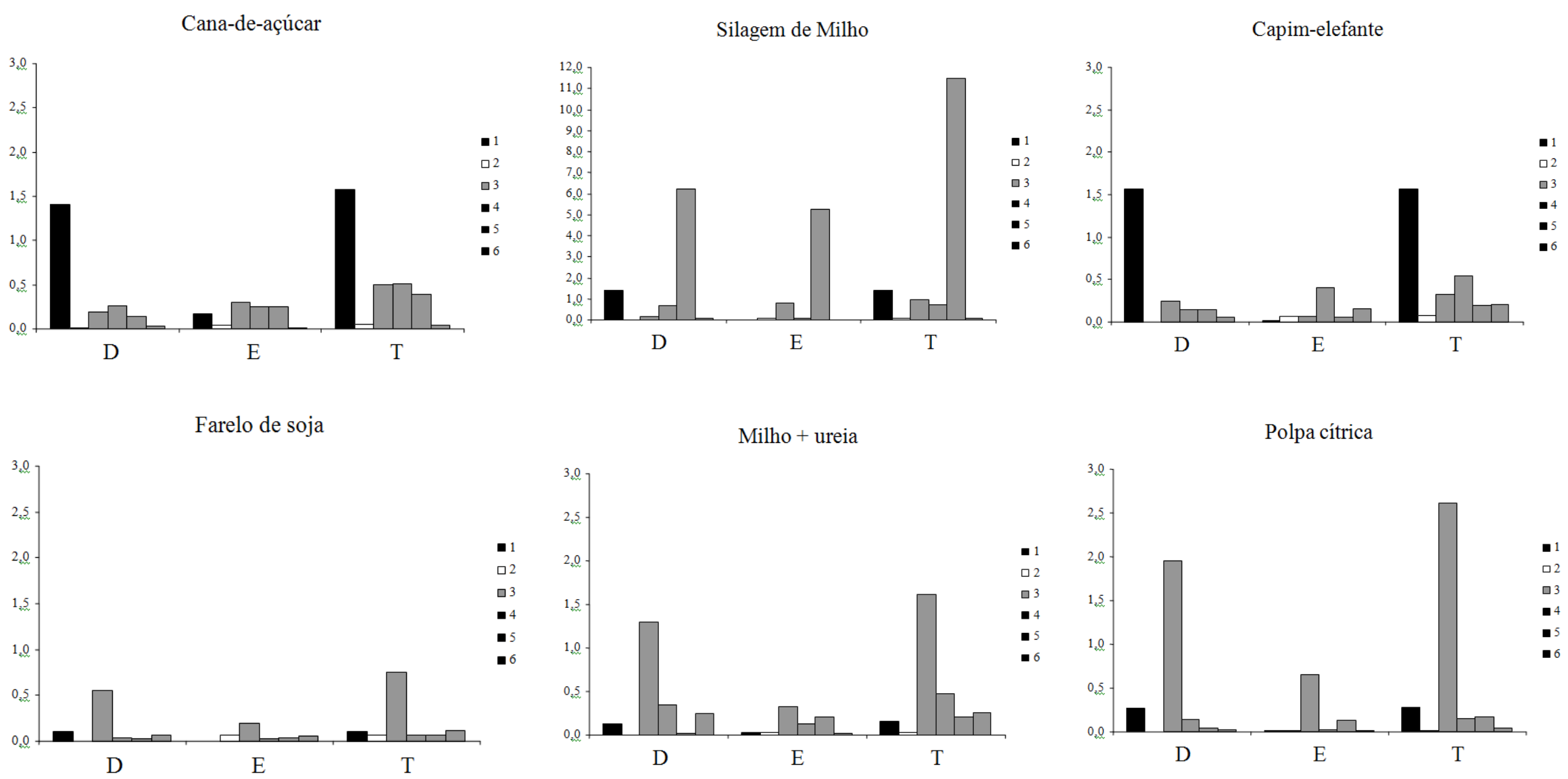

Figura 2. Avaliação das variâncias intra-laboratoriais (D - variância entre dias; E - variância do erro; T - variação aleatória total; T = D + E) 


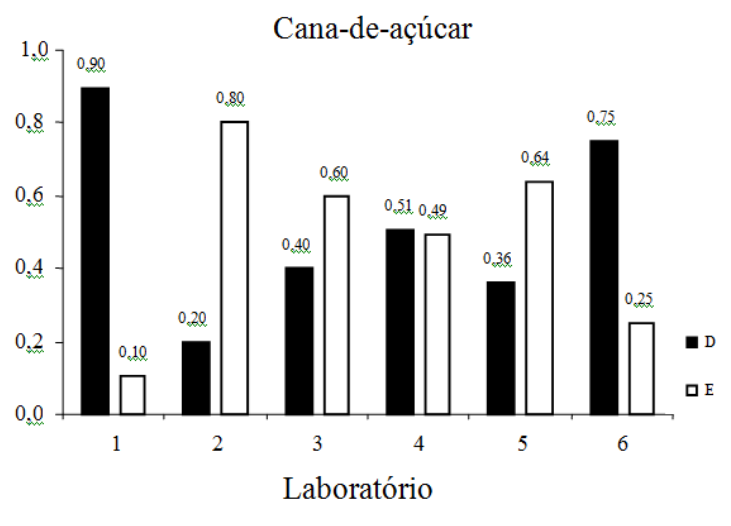

Farelo de soja

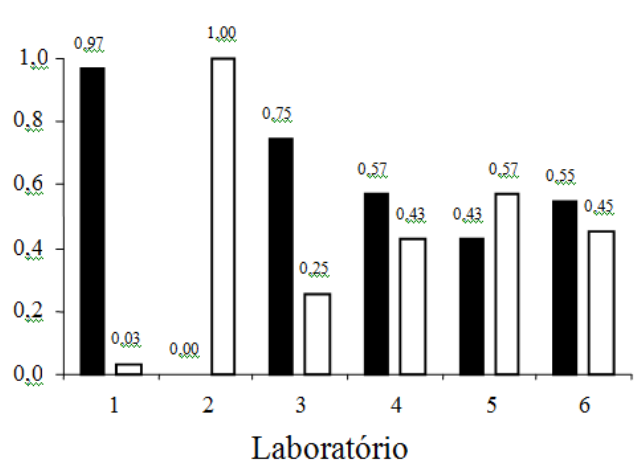

Silagem de milho

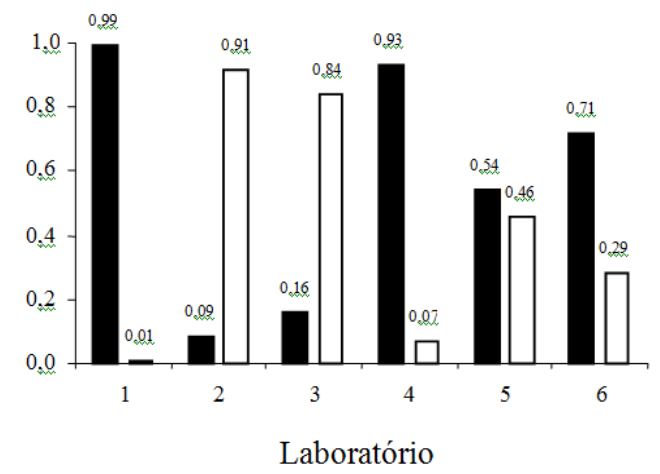

Milho + ureia

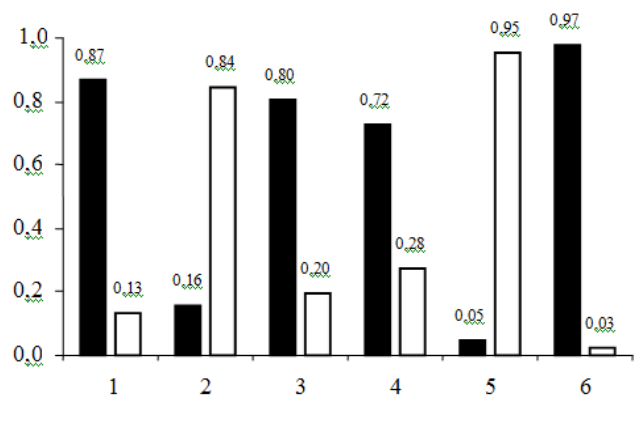

Laboratório
Capim-elefante

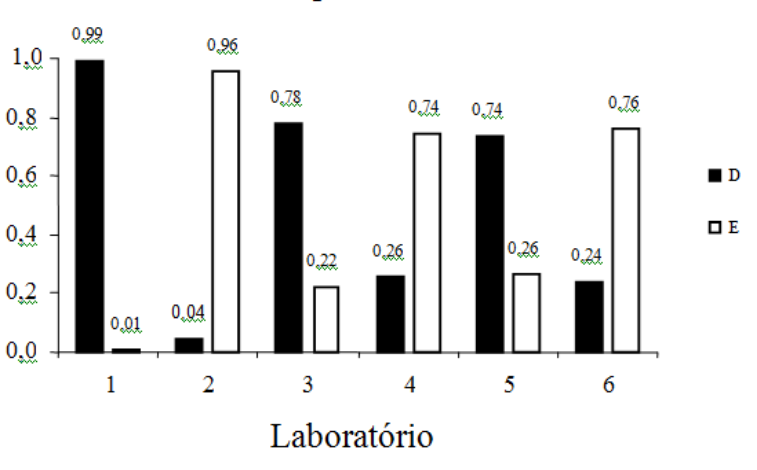

Polpa cítrica

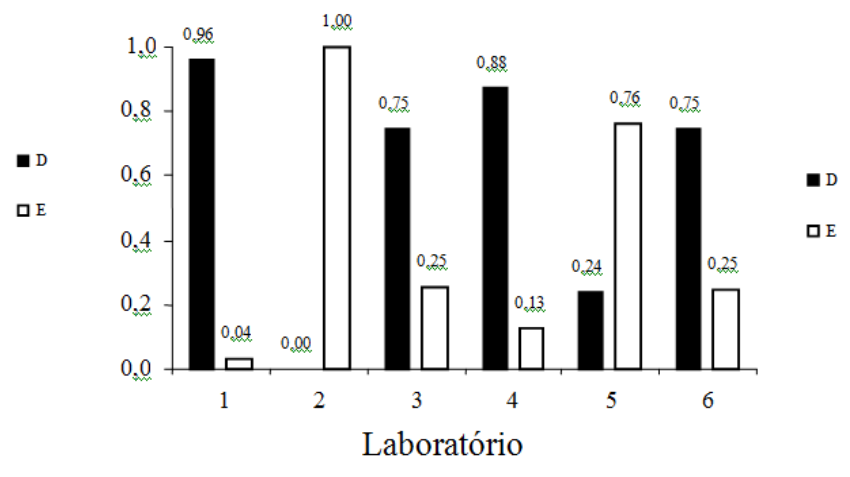

Figura 3. Avaliação fracional da composição da variação aleatória intra-laboratorial (D - contribuição da variância entre dias; E contribuição da variância do erro) 
Rev. Bras. Saúde Prod. Anim., Salvador, v.16, n.3, p.617-631 jul./set.., 2015 http://www.rbspa.ufba.br ISSN 15199940

Tabela 10. Resumo das características quantitativas da avaliação do teor de matéria seca em função dos diferentes laboratórios

\begin{tabular}{cccccc}
\hline \multirow{2}{*}{ Laboratório } & \multicolumn{5}{c}{ Característica } \\
\cline { 2 - 6 } & Peso de alíquota $(\mathrm{g})$ & Temperatura $\left({ }^{\circ} \mathrm{C}\right)$ & Tempo de secagem $(\mathrm{h})$ & $\mathrm{NCM}^{(1)}$ & $\mathrm{TAB}^{(1)}$ \\
\hline 1 & 2 & 105 & 16 & 20 & 30 \\
2 & 2 & 105 & 5 & 20 & nota 2 \\
3 & 3 & 105 & 4 & 36 & 30 \\
4 & 105 & 14 & 18 & 60 \\
5 & 1 & 103 & $15-16$ & 27 & nota 3 \\
6 & 1 & 105 & $7-10$ & 15 & $>60$ \\
\hline
\end{tabular}

${ }^{\mathrm{T}} \mathrm{NCM}=$ número médio de cadinhos manejados por dessecador durantes os procedimentos de rotina nos laboratórios; $\mathrm{TAB}=$ tempo médio de aquecimento da balança pré-pesagem (min).

${ }^{2}$ Assume seguir a recomendação estrita do fabricante da balança.

${ }^{3}$ Aguarda somente o tempo de checagem automática do mecanismo.

Em um dos laboratórios não se observa tempo suficiente para o aquecimento e estabilização dos mecanismos de pesagem (Tabela 10). Em cinco dos seis laboratórios, os pesos pré-secagem são obtidos procedendo-se à dupla tara da balança (peso de recipiente e peso de amostra), procedimento não adequado para quantificação exata de pequenas massas, como na avaliação de ASE. A soma dessas diferenças e/ou procedimentos inadequados pode ter contribuído efetivamente para a baixa reprodutibilidade e mais uma vez evidencia-se que laboratórios diferentes praticam métodos distintos.

Em todos os laboratórios avaliados, as balanças não possuem aferição de acordo com as normas estabelecidas pelo Instituto Nacional de Metrologia (INMETRO), o que aponta possível falta de exatidão nos processos de mensuração.

Conclui-se que os teores de matéria seca estimados pelos laboratórios participantes deste estudo apresentam baixa reprodutibilidade, o que é agravado pela presença de efeito de interação do material avaliado e do laboratório. Os resultados podem induzir falsas diferenças na composição química dos alimentos pela introdução de erros sistemáticos atribuídos à baixa e variada exatidão dos teores de matéria seca.

\section{AGRADECIMENTOS}

Ao INCT-Ciência Animal, ao CNPq e à FAPEMIG (PPM) pelo financiamento da pesquisa.

\section{REFERÊNCIAS}

BARBIN, D. Componentes de variância: teoria e aplicações. 2.ed. Piracicaba: FEALQ, 1993. 120p.

BRUTTEL, P.; SCHLINK, R. Water determination by Karl Fischer titration. Herisau: Metrohm, 2006. 80p.

DETMANN, E.; VALADARES

FILHO, S.C. On the estimation of nonfibrous carbohydrates in feeds and diets. Arquivo Brasileiro de Medicina Veterinária e Zootecnia, v.62, n.4, p.980-984, 2010. 
Rev. Bras. Saúde Prod. Anim., Salvador, v.16, n.3, p.617-631 jul./set.., 2015 http://www.rbspa.ufba.br

HORWITZ, W.A. Evaluation of analytical methods used for regulation of foods and drugs. Analytical

Chemistry, v.54, n.1, p.67A-76A, 1982.

HORWITZ, W.A.; ALBERT, R.;

DEUTSCH, M.J.; THOMPSON, J.N.

Precision parameters of methods of analysis required for nutrition labeling.

Part I. Major nutrients. Journal of

AOAC International, v.73, n.5, p.661680, 1990.

MERTENS, D.R. Challenges in measuring insoluble dietary fiber.

Journal of Animal Science, v.81, n.12, p.3233-3249, 2003.

STATISTICAL ANALYSIS SYSTEM SAS. SAS 9.3 Help and Documentation. Version 9.3. Cary, NC: SAS Institute Inc., 2012.

SILVA, D.J.; QUEIROZ, A.C. Análise de Alimentos: métodos químicos e biológicos. 3 ed. Viçosa: Universidade Federal deViçosa, 2002. 235p.

THIEX, N.; RICHARDSON, C.R. Challenges in measuring moisture content of feeds. Journal of Animal Science, v.81, n.12, p.3255-3266, 2003.

VAN SOEST, P.J.; ROBERTSON, J.B. Analysis of forage and fibrous foods. Ithaca: Cornell University, 1985. 202p.

Data de recebimento: 16/03/2015

Data de aprovação: 25/08/2015 